\title{
Características clínicas de los Pacientes Que Consultan a la clínica de dolor crónico del Hospital Militar Central, HISTÓRICO DE 20 AÑOS
}

\author{
Darío Oliveros MD. .*, Maribel Arrieta MD. ., Elisa Cajigas MD., Edmundo Gonima Valero MD.* \\ ${ }^{1}$ Anestesiólogo Hospital El Tunal, E.S.E. ${ }^{2}$ Anestesióloga, Coordinadora Académica Servicio de Anestesiología, Hospital Militar Central, \\ Profesor asociado Universidad Militar Nueva Granada. ${ }^{3}$ Psicóloga Clínica, Clínica de Dolor Hospital Militar Central. \\ ${ }^{4}$ Anestesiólogo, Algólogo, Coordinador Clínica de dolor Hospital Militar Central, Profesor asociado Universidad Militar Nueva Granada, \\ Universidad de la Sabana
}

\begin{abstract}
Resumen
En este estudio se pretende identificar el perfil demográfico de los pacientes que acuden a la clínica del dolor del Hospital Militar Central que consultan por dolor crónico no maligno, a quienes se les ha valorado de manera integral y multidisciplinaria. Se revisaron los expedientes clínicos de los pacientes atendidos en la clínica de dolor del Hospital Militar Central (HMC) entre abril de 1989 y abril de 2009. Un total de 291 pacientes se tomaron de manera aleatoria simple como muestra representativa para un intervalo de confianza del $95 \%$. Se utilizó un cuestionario que incluyó 26 descriptores que evaluaron datos demográficos, epidemiológicos, respuesta emocional y afectación en áreas de ajuste por la presencia de dolor crónico en estos pacientes. Se encontraron múltiples datos estadísticos, como relevante se halló un perfil de pacientes relacionados con las fuerzas militares en un 90\% (nivel de confianza 95\%, IC 86-93) entre funcionarios militares, no militares y sus beneficiarios. El 49,4\% son militares (nivel de confianza 95\%, IC 43-55\%). Prevalece como entidad más importante el dolor lumbar crónico 41,2\% (nivel de confianza $95 \%$, IC 35-47\%) y el relacionado a miembros superiores e inferiores 11,6\% (nivel de confianza 95\%, IC 7,8 - 15\%). Los diagnósticos más comunes en salud mental dados por psicología o psiquiatría fueron el trastorno de adaptación en el $59 \%$ de los casos (nivel de confianza 95\%, IC 53-64,9\%) y el trastorno de depresión en un 18\% (Nivel de confianza 95\%, IC 14-22,8\%). Se encontró un $81 \%$ de compromiso en el área laboral en pacientes menores de 65 años (nivel de confianza 95\%, IC 76-86\%) por causa del dolor crónico y un 86,3\% (nivel de confianza 95\%, IC 80-92\%) entre aquellos con edades entre los 15 y los 30 años. En conclusión, el dolor crónico como enfermedad de alto costo en la población general, es un factor importante de incapacidad o inhabilidad laboral en los pacientes que consultan a la clínica de dolor del HMC, faltan estudios para definir si la población militar es más susceptible comparada con la población no militar.
\end{abstract}

Palabras clave: clínicas de dolor, dolor, dolor de la región lumbar

\section{CLINICAL CHARACTERISTICS OF PATIENTS CONSULTING CHRONIC PAIN Clinic of Hospital Militar Central, 20-year history}

\begin{abstract}
This study aims to identify the demographic profile of patients who come to the Chronic Pain Clinic of the Hospital Militar Central to consult regarding chronic nonmalignant pain, who have been evaluated in an integral and multidisciplinary way. The medical records of the patients assisted in the Chronic Pain Clinic of the Hospital Militar Central (HMC) between April 1989 and April 2009. A total of 291 patients were randomly selected as a representative sample for a trust interval of $95 \%$. A survey questionnaire was used, which included 26 descriptors evaluating demographic and epidemiological data, and emotional response and affectation in adjustment areas due to the presence of chronic pain in
\end{abstract}

Recibido: Diciembre 2 de 2010 Aceptado: Mayo 15 de 2011

* Correspondencia: Darío Oliveros william.oliveros@gmail.com. Dirección postal: Tr. 3 No. 49-00, Facultas de Medicina, Universidad Militar Nueva Granada, Bogotá, Colombia 
these patients. Multiple statistical data were found. A relevant datum was to find a profile of patients related to the military forces by 90\% (95\% significance level, CI 86-93\%) among military and non-military officers and their beneficiaries. $49.4 \%$ are military personnel (95\% significance level, CI $43-55 \%$ ). Chronic lumbar pain stands out as the most prevalent item - 41.2\% (95\% significance level, CI 35-47\%), and the one related to upper or lower limbs, $11.6 \%$ (95\% significance level, CI 7.8-15\%). Most common diagnoses in mental health provided by the psychology and psychiatry sections were adjustment disorders in 59\% of the cases (95\% significance level, CI 53-64.9\%), and depression disorder at $18 \%$ (95\% significance level, CI 14-22.8\%). An $81 \%$ commitment degree was found in the work force in patients aged less than 65 (95\% significance level, CI 76.65\%) due to chronic pain, and 86.3\% (0.5\% significance level, CI 80-92\%) among those from 15 to 30 years old. To conclude, chronic pain as a high-cost disorder in the general population is an important factor in labor absence leaves or inability among patients consulting at the Chronic Pain Clinic of the HMC. There is a lack of studies to define whether the military population is more susceptible in comparison with the non-military population.

Key words: pain clinics, pain, low back pain

\title{
Características Clínicas dos PaCientes Que CONSUltam Á ClíniCa de dor crônica de Hospital Militar Central, 20 ANOS DE HISTÓRIA
}

\begin{abstract}
Resumo
Este estudo tem como objetivo identificar o perfil demográfico dos pacientes atendidos por uma clínica de dor do Hospital Militar Central com queixa de dor crônica não oncológica, que foram avaliado de uma maneira global e multidisciplinar. Foram revistos os registros clínicos de pacientes atendidos na clínica de dor do Hospital Central Militar (HMC) entre abril de 1989 e abril de 2009. Um total de 291 doentes foram a amostra representativa aleatória simples com um intervalo de confiança de 95\%. Foi usado um questionário que incluía 26 descritores que avaliaram dados demograficos, epidemiológicos, resposta emocional e afetação em áreas de ajuste para a presença de dor crônica nesses pacientes. Encontramos vários dados estatísticos, como relevante foi encontrado um perfil do paciente associadas com os militares em 90\% (nível de confiança de 95\%, IC 86-93) entre os oficiais militares, não militar e seus beneficiários. 49,4\% são militares (nível de confiança de 95\%, IC 43-55\%). Prevalece como entidade mais importante a dor lombar crônica 41,2\% (nível de confiança de 95\%, IC 35-47\%) e com relação aos membros superiores e inferiores 11,6\% (nível de confiança de $95 \%$ CI 7,8-15\%). Os diagnósticos mais comuns em saúde mental dadas pela psicologia ou psiquiatria foram transtorno de adaptação em $59 \%$ dos casos (95\% nível de confiança, IC 53-64,9\%) e transtorno depressivo em 18\% (Nível 95\% de confiança, IC 14 para 22,8\%). Encontramos um 81\% de compromisso na área laboral em pacientes com menos de 65 anos (nível de confiança de 95\%, IC 76-86\%) por causa de dor crônica e 86,3\% (95\% nível de confiança, IC 80 $-92 \%$ ) entre aqueles com idade entre 15 e 30 anos. Em conclusão a dor crônica como uma doença de alto custo na população geral é um fator importante de deficiência ou incapacidade para o trabalho em pacientes que consultam a clínica de dor do HMC, faltam estudos para definir se a população militar é a população mais suscetíveis em comparação com a não militar.
\end{abstract}

Palavras-chave: clínica de dor, dor, dor lombar

\section{Introducción}

El dolor es el principal síntoma por el cual los pacientes acuden a los servicios de urgencias y consulta externa. Se sabe que tiene una prevalencia en Colombia del 53\% (1) y al volverse crónico se convierte en una problemática que produce disfunción a quien la padece. El dolor como experiencia sensorial y psíquica, generalmente debe su aparición a una lesión, que por diversos factores no claramente establecidos, se perpetúa en el tiempo, afectando la calidad de vida de quien la padece, su relación con sus allegados, su desempeño laboral y sus hábitos diarios $(2,3)$.

La prevalencia del dolor crónico en nuestro país no se conoce debido a los sistemas de registro insuficientes. Un 
esfuerzo importante se ha hecho con las diferentes versiones de encuesta nacional del dolor, la última realizada en el marco del quinto estudio nacional del dolor en el 2010, pero ésta y las versiones anteriores se han centrado en estudios poblacionales que no han identificado claramente el espectro crónico del dolor.

Mucho se ha hablado de cuáles podrían ser los factores que modifican una percepción dolorosa hasta convertirla en todo un síndrome de dolor crónico. Pero no es posible dilucidar claramente este epifenómeno si no tenemos claras las bases estadísticas con las que estamos en contacto a diario (4). La prevalencia de dolor crónico varía de estudio en estudio y es dependiente de los estratos culturales atendidos en cada centro de atención para el dolor crónico. A pesar de los más de 25 años de funcionamiento de la clínica de dolor del Hospital Militar (inicio labores en 1984) no conocemos sus características demográficas y tampoco conocemos la prevalencia de las enfermedades manejadas en ella.

En el panorama nacional y local, se requiere una aproximación/caracterización de este tipo de pacientes para favorecer el diseño de estudios que visualicen intervenciones terapéuticas o diagnósticas en esta población de alto costo.

\section{Materiales y métodos}

El estudio se realizó en el hospital de referencia nacional de las fuerzas militares de Colombia, con evaluación de

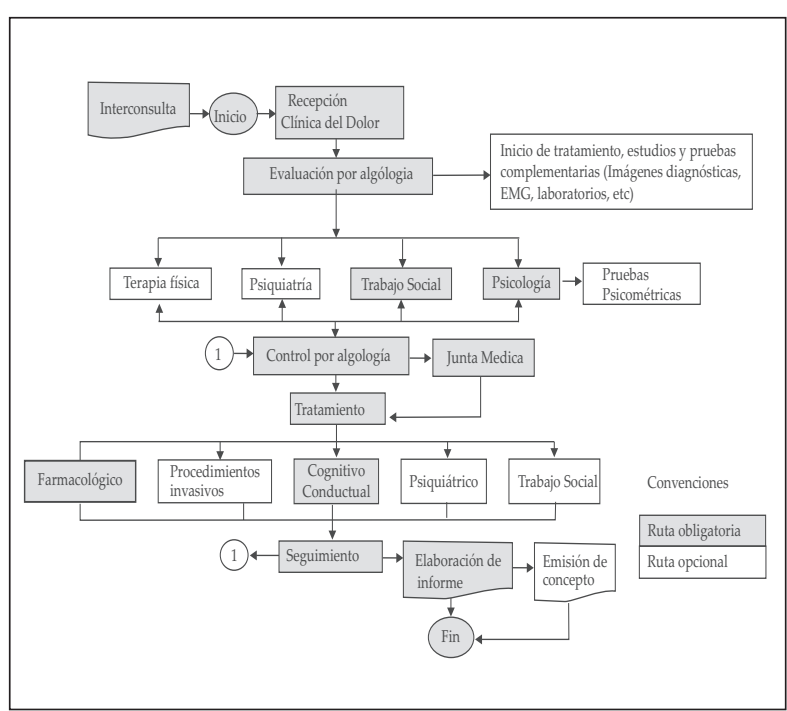

Figura 1. Flujograma organizacional clínica de dolor Hospital Militar Central una muestra representativa de los pacientes que fueron llevados a junta de decisiones de la clínica de dolor crónico durante el periodo de tiempo comprendido entre abril de 1989 y abril de 2009 .

Este es un estudio observacional descriptivo, retrospectivo. En el periodo de estudio se realizaron 630 juntas médicas, reunión multidisciplinaria compuesta como mínimo por anestesiólogo-algesiólogo, psicóloga, psiquiatra de enlace y trabajadora social. Se evaluaron 1768 pacientes de los cuales se tomó una muestra de manera aleatoria simple, seleccionando 291 pacientes, se obtuvieron datos con un intervalo de confianza del $95 \%$ y una p de 0,05 . Se utilizó como herramienta de cálculo la disponible en la dirección en internet. http:// www.feedbacknetworks.com/cas/experiencia/ sol-preguntar-calcular.htm.

La dinámica de la clínica de dolor es compatible con una clínica de dolor multidisciplinaria (figura 1) (5). Se incluyeron para el análisis de datos solamente aquellos pacientes que recibieron atención médica en la clínica del dolor del Hospital Militar Central con dolor crónico no oncológico, que fueron evaluados de manera multidisciplinaria en junta de decisiones. Los criterios de inclusión entonces son: pacientes con dolor por más de tres meses en donde se compruebe que secundario al dolor existen alteraciones emocionales secundarias al dolor o afección en áreas de ajuste y concuerden a un dolor de origen somático, neuropático, visceral, psicógeno o mixto cuyo origen no sea oncológico. Este perfil es el que se utiliza en la clínica para definir que el paciente tiene dolor crónico y se beneficia de ingresar al programa. Se excluyeron los pacientes que no tenían evaluación completa o que no cumplieron los criterios de inclusión (e.g pacientes sin dolor crónico, Herpes zoster, cáncer, pacientes con historia o valoración incompleta).

Se buscaron las actas del servicio en el que constan en su totalidad el número de pacientes valorados en el lapso de tiempo estudiado. Se les asignó un número y posteriormente se hizo un muestreo aleatorio simple. Se tomó al dolor lumbar crónico como referencia para el cálculo de prevalencias. Por ser la entidad más frecuentemente atendida en el servicio con una estimación porcentual a priori del $35 \%$, se utilizó como distribución de la respuesta en el cálculo de muestra.

Para recolectar la información se utilizó un instrumento diseñado para tal fin (Anexo A). Este instrumento fue llenado por el investigador principal y se aplicó a las historias de los casos seleccionados manualmente. 
En las historias clínicas seleccionadas se aplicó el instrumento de recolección de la información, a la consulta de ingreso, a cada uno de los controles y a lo consignado en la junta de decisiones. Los datos recolectados fueron introducidos y evaluados en una base de datos de EXCEL versión 2007 utilizando las tablas dinámicas del mismo programa. Una vez completada la recolección y depuración de datos, se analizaron mediante tablas de frecuencias y su distribución. Los cálculos estadísticos se realizaron con ayuda del software Epidat 3,1 (junta de Galicia y Organización Panamericana de la Salud). No se realizaron pruebas de significancia estadística pues no se propuso encontrar asociaciones en los datos recolectados por el tipo de diseño del estudio.

Esta investigación se clasificó como Investigación sin riesgo según lo establecido en la resolución № 008430 de 1993 (4 de octubre de 1993) Del Ministerio de Salud, República de Colombia. Se protegió la privacidad del individuo, sujeto de investigación, sin identificarlo pues el diseño del estudio no lo requirió.

\section{Resultados}

Las edades de los pacientes oscilaron entre los $15 \mathrm{y}$ los 80 años. El promedio de edad en el grupo fue de 46 años, con una desviación estándar de 17 años, Para el análisis los pacientes se dividieron en tres grupos de acuerdo a la edad: pacientes entre 15 y 30 años, entre 31 y 65 años y mayores de 65 años. $\mathrm{Al}$ analizar los datos se encontró que el grupo más representativo es el de menores de 30 años, $45 \%$ (nivel de confianza 95\%, IC 40-51\%) (Tabla 1). El 67\% de pacientes fueron hombres (nivel de confianza 95\%, IC $62,5-73,5)$, la mayor parte de ellos militares $n=140$, casados con un bajo nivel de escolaridad (secundaria incompleta o primaria completa o incompleta), con afectación en área laboral por la presencia de dolor crónico (Tablas 1 y 2).

El origen del dolor se relacionó en un $48 \%$ con un evento traumático no quirúrgico (nivel de confianza 95\%, IC 41,8$53,6 \%$ ), de estos pacientes el $57,7 \%$ refiere un inicio súbito del dolor (nivel de confianza 95\%, IC 51,8-63,5\%), con características somáticas y localizado más frecuentemente en la zona lumbar baja. El tipo de dolor somático, visceral y neuropático se presenta en un 70\%, $2 \%$ y $28 \%$ respectivamente (Tabla 1).

La intensidad del dolor en el momento de la primera consulta fue mayor a siete en la escala visual análoga (EVA) del dolor en el $66 \%$ de los pacientes; el $26 \%$ refirió dolor entre 5 - 8 y el $5 \%$ de los pacientes refirieron dolor menor a cinco. El porcentaje restante de pacientes no entendie-
Tabla 1. Características socio demográficas de pacientes que asisten a la clínica de dolor crónico

\begin{tabular}{|c|c|c|}
\hline VARIABLE & $\mathbf{N}$ & $\%$ \\
\hline $\begin{array}{l}\text { Genero } \\
\text { Masculino } \\
\text { Femenino }\end{array}$ & $\begin{array}{c}98 \\
198\end{array}$ & $\begin{array}{l}34 \\
66\end{array}$ \\
\hline $\begin{array}{l}\text { EPS } \\
\text { Ejercito } \\
\text { Armada } \\
\text { Fuerza Aérea } \\
\text { HOMIC } \\
\text { Otro }\end{array}$ & $\begin{array}{l}204 \\
15 \\
35 \\
12 \\
25\end{array}$ & $\begin{array}{c}70 \\
5 \\
12 \\
4 \\
9\end{array}$ \\
\hline $\begin{array}{l}\text { Estado Civil } \\
\text { Casado } \\
\text { Soltero } \\
\text { Viudo } \\
\text { Divorciado } \\
\text { Unión Libre } \\
\text { No hay dato }\end{array}$ & $\begin{array}{c}127 \\
93 \\
15 \\
9 \\
46 \\
1\end{array}$ & $\begin{array}{c}43,6 \\
32 \\
5 \\
3 \\
16 \\
0,34\end{array}$ \\
\hline $\begin{array}{l}\text { Nivel Educativo } \\
\text { Técnico } \\
\text { Universitario } \\
\text { Primaria Incompleta } \\
\text { Primaria Completa } \\
\text { Secundaria Completa } \\
\text { Secundaria Incompleta } \\
\text { Analfabeta } \\
\text { Escolaridad baja* } \\
\text { No hay dato }\end{array}$ & $\begin{array}{c}9 \\
28 \\
30 \\
49 \\
75 \\
96 \\
2 \\
177 \\
2 \\
\end{array}$ & $\begin{array}{c}3 \\
9 \\
10 \\
17 \\
26 \\
33 \\
1 \\
61 \\
1\end{array}$ \\
\hline $\begin{array}{l}\text { Tipo de dolor } \\
\text { Somático } \\
\text { Visceral } \\
\text { Neuropatico }\end{array}$ & $\begin{array}{c}204 \\
6 \\
81\end{array}$ & $\begin{array}{c}80 \\
2 \\
28\end{array}$ \\
\hline $\begin{array}{l}\text { Edad por grupos } \\
\text { Entre } 18 \text { y } 30 \text { años } \\
\text { Entre } 31 \text { y } 65 \text { años } \\
\text { Mayores de } 65 \text { años }\end{array}$ & $\begin{array}{c}132 \\
128 \\
31\end{array}$ & $\begin{array}{l}45 \\
44 \\
11\end{array}$ \\
\hline $\begin{array}{l}\text { Ocupación } \\
\text { Retirado/Pensionado } \\
\text { Militar activo } \\
\text { No relacionado a fuerzas militares o } \\
\text { Beneficiario } \\
\text { Desempleado }\end{array}$ & $\begin{array}{c}36 \\
144 \\
75 \\
36\end{array}$ & $\begin{array}{l}12 \\
50 \\
26 \\
12\end{array}$ \\
\hline $\begin{array}{l}\text { Presenta otra enfermedad crónica } \\
\mathrm{Si} \\
\mathrm{No}\end{array}$ & $\begin{array}{c}49 \\
242\end{array}$ & $\begin{array}{l}17 \\
83\end{array}$ \\
\hline $\begin{array}{l}\text { Tipos de lesión asociados a dolor crónico } \\
\text { Cortante } \\
\text { Punzante } \\
\text { Corto contundente } \\
\text { Contuso } \\
\text { Corto punzante } \\
\text { Arma de Fuego } \\
\text { Medios físico/térmicos } \\
\text { Agentes químicos } \\
\text { Agentes Biológicos } \\
\text { Psicológicos } \\
\text { No Hay dato }\end{array}$ & $\begin{array}{c}31 \\
3 \\
3 \\
89 \\
4 \\
39 \\
2 \\
0 \\
3 \\
9 \\
108 \\
\end{array}$ & $\begin{array}{c}11 \\
1 \\
1 \\
31 \\
1,3 \\
13,4 \\
0,6 \\
0 \\
1 \\
3 \\
37 \\
\end{array}$ \\
\hline $\begin{array}{l}\text { Origen del dolor } \\
\text { No traumático } \\
\text { Traumático no post quirúrgico } \\
\text { Traumático Post quirúrgico }\end{array}$ & $\begin{array}{c}109 \\
139 \\
43\end{array}$ & $\begin{array}{l}37 \\
48 \\
15\end{array}$ \\
\hline
\end{tabular}




\begin{tabular}{lcc}
\hline Inicio de dolor & & \\
Súbito & 168 & 58 \\
Gradual & 123 & 42 \\
\hline EVA en primera consulta & & \\
$2-4$ & 14 & 5 \\
$5-7$ & 75 & 26 \\
$8-10$ & 192 & 66 \\
NO ENTIENDEN PREGUNTA & 8 & 2 \\
NO HAY DATO & 2 & 1 \\
\hline
\end{tabular}

*Escolaridad baja: suma de pacientes con bachillerato incompleto, primaria incompleta o completa y sin educación alguna ++Paciente en edad productiva se considero todo aquel entre los 15 y 65 años

ron la escala de evaluación o no se consignó en la historia clínica el dato (Tabla 1). El diagnóstico más frecuente fue dolor lumbar crónico, seguido por el dolor crónico de extremidades. En la Tabla 3 se enumeran las primeros cinco motivos de consulta en algología, su prevalencia e intervalos de confianza y en la Tabla 4 los diagnósticos principales de la evaluación por psicología, psiquiatría y trabajo social.

El dolor crónico impacta en la población estudiada en las áreas de ajuste ocupacional/laboral, social, familiar, afectiva y sexual en un $78 \%, 66 \%, 13,4 \%, 6 \%$ y $45 \%$ respectivamente (los valores no suman $100 \%$ pues en cada paciente están afectadas en promedio dos áreas de ajuste con una desviación estándar de 1,11) (Figura 2). La esfera más alterada por el cuadro de dolor fue la laboral en un $78 \%$ (nivel de confianza 95\%, IC 73,4-83\%), el área sexual y social también fueron importantes (Tabla 2). La alteración psicofisiológica más común fue la alteración del patrón del sueño en un $65 \%$ (nivel de confianza 95\%, IC 59,3-70\%). Como conducta des-adaptativa relacionada con la presencia de dolor se encontró en mayor proporción el abandono de responsabilidades 22,3\% (nivel de confianza 95\% IC 17,4-27\%). En un $72 \%$ de los casos (nivel de

confianza 95\%, IC 67-77\%) no se encontró un estado emocional primario previo al dolor crónico, pero en los pacientes en quienes si hay un estado emocional primario previo al dolor, se encontró como predominante los estados depresivos en un $12,4 \%$ ( nivel de confianza $95 \%$, IC 8,4-16\%) (Tabla 2).

En cuanto a respuestas emocionales secundarias se encontró que el 95\% de los pacientes presentan como conducta dolorosa más frecuente un cambio emocional, la irritabilidad en un 58\% (nivel de confianza 95\%, IC: 52-63,5\%) de los casos, seguida por la preocupación y la tristeza. En
Tabla 2. Descriptores de valoración Psicología, Trabajo social, Psiquiatría de enlace ( $\mathrm{n}=291$, cada ítem puede responderse de manera múltiple, por lo tanto no es porcentual la forma en que se muestran los datos)

\begin{tabular}{lc}
\hline \multicolumn{1}{c}{ VARIABLE } & $\mathbf{n}$ \\
\hline Alteración Psico- fisiológica & \\
Sueño & 213 \\
Cambios en alimentación (aumento o disminución en ingesta) & 36 \\
Mareos & 21 \\
Nauseas & 23 \\
Vomito & 2 \\
Pérdida de peso & 5 \\
Sudoración & 11 \\
Diarrea & 0 \\
Cefalea & 52 \\
\hline
\end{tabular}

Componente emocional Primario

Depresión $\quad 47$

Ansiedad $\quad 29$

Rasgos mal adaptativos de personalidad $\quad 8$

Stress post traumático $\quad 8$

Ninguno 213

Respuesta emocional secundaria

$\begin{array}{ll}\text { Irritabilidad } & 168\end{array}$

Angustia $\quad 49$

Preocupación 139

Aislamiento $\quad 29$

$\begin{array}{ll}\text { Apatía } & 13\end{array}$

Tristeza 130

Ansiedad $\quad 41$

Temor 19

No tiene 14

Conductas des-adaptativas

Abuso de medicamentos $\quad 5$

Inactividad $\quad 75$

Abandono de responsabilidades $\quad 26$

$\begin{array}{ll}\text { No Reportada } & 195\end{array}$

Otros factores asociados a la percepción del dolor

Duelo mal elaborado $\quad 23$

Disfunción de Pareja $\quad 50$

Disfunción Familiar $\quad 42$

Ausencia de apoyo familiar percibido $\quad 27$

Antecedente de maltrato en la infancia $\quad 20$

Antecedente de abandono en la infancia 6

Sobrecarga de responsabilidades en la infancia $\quad 5$

Refuerzo familiar $\quad 14$

No tiene 156

Otro 9

\begin{tabular}{lc}
\hline Alteración en áreas de ajuste & \\
Laboral & 228 \\
Social & 192 \\
Familiar & 39 \\
Afectivo & 17 \\
Sexual & 123 \\
Ninguno & 30 \\
\hline
\end{tabular}


varias ocasiones se encuentra una mezcla de emociones en el mismo paciente (Tabla 2).

Se determinó la proporción de pacientes con dolor lumbar crónico y afección en el área laboral, encontrándose que el $86 \%$ de los diagnosticados con esta entidad tienen compromiso en el área laboral y solo un $14 \%$ no lo tienen. (Figura 3). En los pacientes con dolor lumbar crónico y compromiso laboral se encontró una mayor proporción de afectados en el grupo de militares $95 \%$ comparado con los no militares 75\% (Figuras 4 y 5 ).

Tabla 3. Principales diagnósticos en junta de decisiones por algologia $\mathrm{n}=291$

\begin{tabular}{lll}
\hline \multicolumn{1}{c}{ Diagnóstico } & \multicolumn{2}{c}{ Prevalencia estimada (IC) } \\
\hline Dolor lumbar crónico & $41.2 \%$ & $(35.4-47 \%)$ \\
M796 & $12 \%$ & $(7.8-15.5 \%)$ \\
Sindrome doloroso regional & $9.9 \%$ & $(6.3-13.5 \%)$ \\
complejo & $9.9 \%$ & $(6.3-13.5 \%)$ \\
Sindrome miofascial & $6 \%$ & $(2.9-8.7)$ \\
Dolor testicular &
\end{tabular}

IC: Intervalos de confianza. M796 Dolor en miembro.

Solo se muestran los cinco primeros diagnósticos

Tabla 4. Principales diagnósticos en junta de decisiones por psicología $\mathrm{n}=291$

\begin{tabular}{|c|c|c|}
\hline \multirow{2}{*}{$\begin{array}{l}\text { Diagnóstico } \\
\text { Trastornos de adaptación }\end{array}$} & \multicolumn{2}{|c|}{$\begin{array}{c}\text { Prevalencia } \\
\text { estimada (IC) }\end{array}$} \\
\hline & $59 \%$ & $(53 .-64.9 \%)$ \\
\hline Otros trastornos depresivos recurrentes & $18 \%$ & $(14-22.8 \%)$ \\
\hline Otros trastornos de ansiedad & $6 \%$ & $(3-8.7 \%)$ \\
\hline Trastorno mixto de ansiedad y depresión & $5 \%$ & $(2.4-7.8 \%)$ \\
\hline $\begin{array}{l}\text { Problemas relacionados entre esposos o } \\
\text { pareja }\end{array}$ & $4 \%$ & $(2-6.5 \%)$ \\
\hline
\end{tabular}

IC: Intervalos de confianza. M796 Dolor en miembro.

Solo se muestran los cinco primeros diagnósticos y sexual. los porcentajes no suman $100 \%$ pues en promedio hay afectacion de dos áreas de ajuste por paciente como parte de la conducta dolorosa en el sindrome de dolor crónico, en su gran mayoria afectacion en area laboral y social.

Al hallar un compromiso en el área laboral tan importante $78 \%$ ( $n=228$, nivel de confianza $95 \%$, IC 73,4-83\%) y sabiendo que los pacientes en edad productiva son quienes tienen una participación económica activa en las sociedades, se muestra el porcentaje de pacientes que siendo menores de 65 años y no teniendo ninguna otra enfermedad crónica diferente al dolor alteran su vida laboral por la persistencia del síndrome doloroso. Es importante notar en la población estudiada que los pacientes del grupo que tienen más de 15 años de edad pero menos de 30 años tienen compromiso del área laboral como conducta dolorosa en respuesta al síndrome de dolor crónico en un gran porcentaje ( $86 \%$ de pacientes en estas edades, nivel de confianza $95 \%$, IC $80-92 \%$ edad media de 24 años, desviación estándar de 4,96 años). En la figura 6 se muestran solamente aquellos pacientes que tienen edad productiva y tienen afección en el área laboral n=211, Se subdivide en grupos de pacientes sin otras enfermedades crónicas (entre 15 y 30 años o mayores a 30 años y menores de 65 años) y quienes tienen alguna comorbilidad.

\section{Sesgos}

Los datos obtenidos en el estudio deben interpretarse en el contexto de un hospital con características particulares. El Hospital Militar Central atiende solamente al personal perteneciente a las Fuerzas Militares de Colombia y sus beneficiarios, razón por la cual vale la pena reproducir este estudio en clínicas de dolor crónico multidisciplinarias en donde se atienda a la población en general.

Consideramos que al querer identificar el perfil epidemiológico de la clínica de dolor del Hospital Militar Central se nos facilitó la selección de la muestra y los sujetos de estudio sin encontrarse sesgos en este proceso pues el archivo utilizado es organizado y las actas de cada una de las juntas de decisiones se encuentran debidamente archivadas.

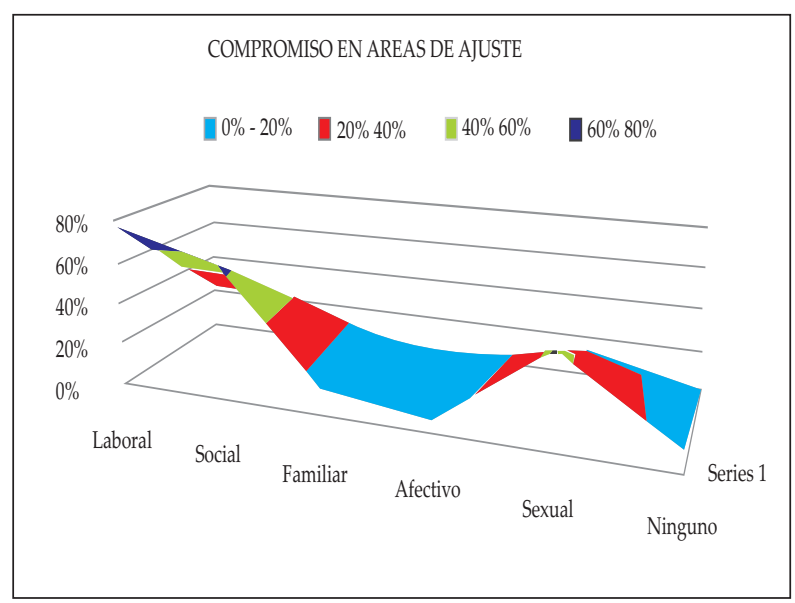

Figura 2. Compromiso en áreas de ajuste. Predominio de áreas laboral, social 


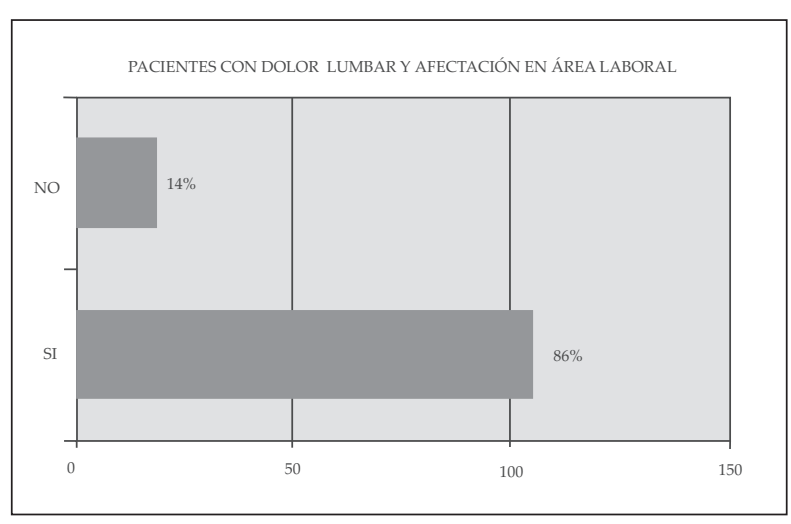

Figura 3. Del número total de pacientes con dolor lumbar crónico n=120, el $86 \%$ tienen afectacion en el área laboral $n=103$.

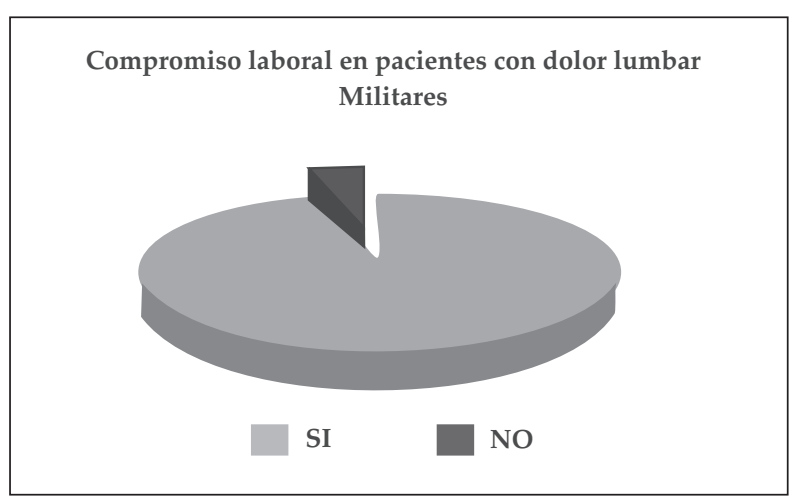

Figura 4. Militares con dolor lumbar crónico n= 65 y compromiso del área laboral $\mathrm{n}=62$.

Para controlar la recolección de la información, se hizo de forma manual por un único investigador y así eliminar un posible sesgo por error de clasificación diferencial debido al entrevistador, en sesiones programadas de no más de seis horas día, con un promedio de revisión por historia clínica de 20 minutos, tiempo adecuado para leer en su totalidad la historia para poder completar los ítems del formato de recolección de datos.

\section{Discusión y análisis}

Según datos reportados por la Asociación Colombiana para el estudio del dolor (ACED), solamente un 15\% de la población Colombiana con dolor consulta a clínicas especializadas (6); y en la última encuesta nacional de dolor, quinta versión, se reportó un $21 \%$ de prevalencia de dolor presente por más de seis meses en una población de 9'666.032. Sin embargo, la prevalencia específicamente del dolor crónico en la población colombiana no se conoce.

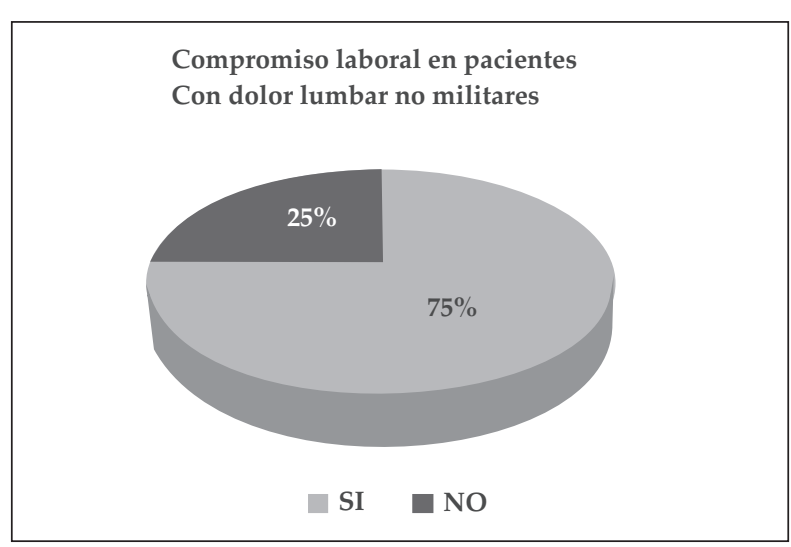

Figura 5. No militares con dolor lumbar crónico $\mathrm{n}=55$ y compromiso del área Laboral $\mathrm{n}=41$

Los pacientes atendidos en la clínica de dolor del HOMIC son en su gran mayoría militares, funcionarios relacionados a las fuerzas militares sin tener carrera militar o beneficiarios de estos (familiares), la proporción de pacientes atendidos como particulares es mínima y no siempre son pacientes con dolor crónico no maligno. En este estudio se determinó que se atiende una gran cantidad de pacientes con diversas entidades patológicas sobresaliendo la alta prevalencia de dolor lumbar crónico.

La interpretación de los resultados de los estudios epidemiológicos sobre dolor lumbar es un reto por la inconsistencia y la variabilidad de criterios utilizados para definir el dolor de espalda y la falta de estandarización de los mismos. En general se acepta que un 70-80\% de la población adulta de las sociedades occidentales tendrá alguna vez en su periodo de vida dolor lumbar, pero

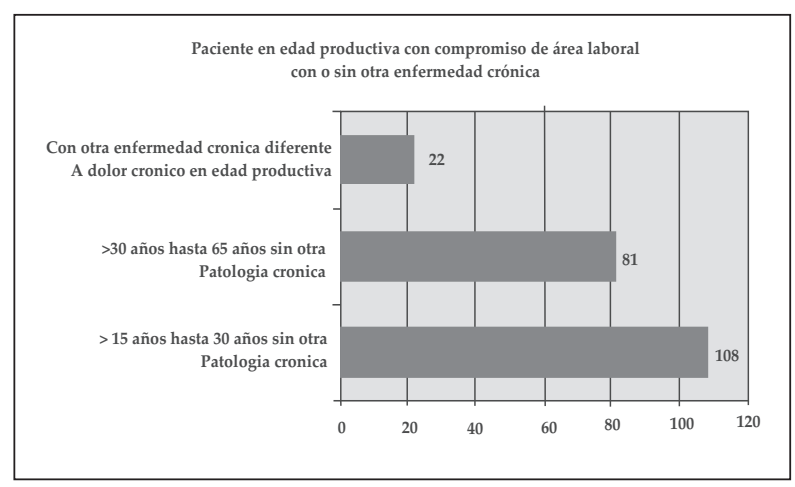

Figura 6. Pacientes en edad productiva y compromiso laboral $\mathrm{n}=211 \mathrm{sin}$ otra enfermedad crónica $n=189$ (menor a 30 años $n=108$ entre 30 y 60 años $\mathrm{n}=81$ ) o con otra enfermedad crónica $n=22$ 
solamente un $7 \%$ lo tendrá por más de doce semanas $(7,8)$. En la población estudiada encontramos una prevalencia para dolor lumbar crónico del $41 \%$, cifra alarmante dada la repercusión laboral y económica que ha caracterizado esta enfermedad.

El dolor de extremidades fue la segunda entidad más frecuente, dato que no representa a pacientes heridos en combate, pues solamente un $38 \%$ de los diagnosticados con dolor en extremidades o con Síndrome de dolor regional complejo tienen una lesión causada por arma de fuego, por una mina, por una granada o cilindro. El trauma contuso de extremidades es aun más importante que el causado por arma de fuego en los pacientes con dolor en extremidades; sin embargo, si la presentación clínica del síndrome doloroso es neuropático, las heridas de guerra toman preponderancia, como se encontró en una observación previa realizada en heridos en combate (9).

Del componente psicológico del síndrome doloroso, el trastorno de adaptación, como diagnóstico principal en las juntas de decisiones, representa la respuesta des-adaptativa y excesiva al estresor enmarcada en la vivencia de dolor por más de doce semanas con una incidencia superior a la esperada en estos pacientes ( $20 \%$ vs $59 \%$ ). En general se dice que un $20 \%$ de quienes están expuestos a estresantes que se perpetúan en el tiempo, sufrirán de este trastorno (10). En la muestra escogida se encontró una prevalencia alta que podría estar influenciada por factores culturales, el grupo de edad, el oficio, significado-experiencia del estresor o un conjunto de estos. Un dato preocupante es que esta entidad se acompaña de deterioro significativo de la actividad social y laboral claramente evidenciada en la descripción aquí mostrada; lo más grave es que considerando el trastorno de adaptación como entidad única se sabe que su resolución depende de la desaparición del estresante (10), poco probable para la mayoría de los dolores crónicos.

La conducta dolorosa se puede valorar de manera somera al determinar el compromiso en áreas de ajuste de cada individuo. Con tan alta prevalencia del trastorno de adaptación se espera un compromiso importante en actividades social y laboral (11).

\section{Dolor lumbar y situación laboral}

No hay estimativos poblacionales de dolor lumbar crónico en Latino América y en general, no hay políticas gubernamentales que faciliten la atención especializada de esta entidad en el territorio nacional. El dolor lumbar crónico tiene un impacto social alto en el mundo industrializado; en los Estados Unidos el dolor lumbar es la segunda causa de ausentismo laboral (12-14). Aunque no es posible determinar datos epidemiológicos con el presente estudio, sí se destaca el compromiso laboral en pacientes con dolor lumbar y sugiere una prevalencia más alta en el personal militar, que habría que corroborar con un estudio posterior.

En el presente estudio se encontró que el 78\% de los pacientes tienen repercusión en el área laboral por consecuencia directa de su enfermedad. En estudios colombianos se promedia un $49,8 \%$ de repercusión media o alta en el área laboral por presencia de dolor (sin puntualizarse si es crónico o no). El costo estimado en este contexto pagado por empleadores y EPS, debido a las incapacidades por estos dolores, es del orden de 23 mil millones de pesos (6). Un programa de prevención o rehabilitación en este grupo de pacientes podría ser costo efectivo pero no es posible soportar su impacto a priori por la falta de datos precisos.

El nivel socioeconómico de los pacientes no se pudo establecer porque solamente se encontró esta información en el $8 \%$ de las historias. Por otra parte, la evaluación del impacto en la mejoría del paciente en funcionalidad, calidad de vida, reinserción laboral, estabilización afectiva, disminución del dolor y el sufrimiento, no se determinó con los datos obtenidos ya que el EVA post tratamiento solo se encontró anotado en el $22 \%$ de las historias y no se utilizan escalas de calidad de vida ni de otros parámetros mencionados, al finalizar el tratamiento.

Finalmente, para próximas investigaciones se recomienda seguir un formato actualizado estándar y diligenciar todos los ítems allí solicitados. Los diagnósticos deben diligenciarse según las guías actuales de la IASP y codificarse con la nomenclatura aceptada de manera internacional.

\section{Conclusión}

El dolor crónico como enfermedad de alto costo en la población general es un factor importante de incapacidad o inhabilidad laboral en los pacientes que consultan a la clínica de dolor del HOMIC. En este estudio se plantea la relación entre dolor lumbar, ser militar y la repercusión laboral correspondiente, sin poder asumir una relación causal o relación alguna entre estas variables, se debe contrastar posteriormente en estudios de diseño analítico.

Se sugiere que es relevante iniciar programas de prevención primaria en dolor lumbar y es imperativo implementar estrategias para evaluar y documentar el impacto de la atención que se brinda en la clínica, por lo cual se propone: 
1. La utilización de un formato único para los pacientes que son llevados a junta Médica teniendo en cuenta varios ítems utilizados en el instrumento de recolección de datos de este artículo.

2. La implementación de escalas validadas de evaluación en calidad de vida como la SF-36, HAD, PGWB al iniciar y terminar tratamiento o continuar utilizando el índice de Lattinen.

3. En cada consulta de control y la última cita en la clínica debe fortalecerse el registro de preguntas mínimas: el EVA, el lattinen, el consumo actual de medicamentos con nombres y dosis, si se han suministrado los medicamentos formulados, si ha sido posible seguir el tratamiento y la mejoría global expresada en términos de porcentaje.

\section{Referencias}

1. Asociación colombiana para el estudio del dolor aced. Cuarta encuesta nacional de dolor 2008. Texto online: $\underline{\mathrm{http}: / / \text { dolor.Org.Co/ }}$ index.Php?Option=com content\&view=article\&id=69\&itemid=84

2. Smith b, chambers $w$, smith c. The impact of chronic pain in the community. Family practice. 2001;18:292-299.

3. Elliott $\mathrm{a}$, smith $\mathrm{b}$, penny $\mathrm{k}$, et al. The epidemiology of chronic pain in the community. Lancet. 1999;354: 1248-52.Eriksen j, jensen m, sjøgren $\mathrm{p}$ et al. Epidemiology of chronic non-malignant pain in denmark. Pain. 2003;106:221-228.

4. Eriksen $j$, jensen $m$, sjøgren $p$ et al. Epidemiology of chronic nonmalignant pain in denmark. Pain. 2003;106:221-228.

5. Ibañez j. Situación actual del tratamiento del dolor crónico en españa. Rev. Esp. Anestesiol. Reanim. 2005; 52:127-130

6. Boswell $\mathrm{m}$. Weiner's pain management, seventh edition, assuring the quality of pain services: assessing outcomes, chapter 9 , taylor \& francis, new york, 2006

7. Breivick $h$, collett $b$, ventafridda $v$, et al. Survey of chronic pain in europe: prevalence, impact on daily life, and treatment. Eur $j$ pain 2006; 10:287-333.
8. Bassols a, bosch $\mathrm{f}$, campillo m, et al. An epidemiological comparison of pain complaints in the general population of catalonia (spain). Pain 1999; 83:9-16.

9. Harstall c, ospina m. How prevalent is chronic pain? Pain clin. Updates 2003; 11(2):1-4.

10. Asociación colombiana para el estudio del dolor aced. Quinto estudio nacional del dolor 2010.Texto on line: http://dolor.Org.Co/ index.Php?Option=com content\&view=article\&id=69\&itemid $=84$

11. Loeser jd. Bonica's management of pain third edition, general considerations of chronic pain, chapter 10, lippincott williams \& wilkins publishers, seattle, washington, 2001.

12. Jay gw. Practical guide to chronic pain syndromes, part ii soft tissue pain syndromes, pfizer, inc. Informa healthcare usa, new york, 2010. Pág. 115-144.

13. Torrance $\mathrm{n}$, smith $\mathrm{b}$, bennett $\mathrm{m}$, lee $\mathrm{a}$. The epidemiology of chronic pain of predominantly neuropathic origin. Results from a general population survey. The journal of pain 2006; 7(4):281-289.

14. Lewandowsky w. Psychological factors in chronic pain: a worthwhile undertaking for nursing? Archives of psychiatric nursing 2004; 18(3):97-105.

15. Penzo w. El dolor crónico, aspectos psicológicos, biblioteca de psicología, psiquiatría y salud serie salud 2000, ediciones martínez roca, barcelona-españa, 2000.

16. Gullacksen ac, lidbeckj. The life adjustment process in chronic pain: phychosocial assessment and clinical implications. Pain res manage 2004; 9(3): 145-153.

17. Tunks er, weir $\mathrm{r}$, crook $j$. Epidemiologic perspective on chronic pain treatment 2008;53(4):235-242.

18. Dickens c, jayson $m$, creed f. Psychological correlates of pain behavior in patients with chronic low back pain. Psychosomatics 2002; 43:42-48.

19. Becker $\mathrm{n}$, bondegaard $\mathrm{t}$, kornelius a, et al. Pain epidemiology and health related quality of life in chronic non-malignant pain patients referred to a danish multidisciplinary pain center. Pain 1997; 73:393-400

20. Mccracken $1 \mathrm{~m}$, evon d, karapas et. Satisfaction with treatment for chronic pain in a specialty service: preliminary prospective results. Eur j pain 2002; 6: 387-393. 


\section{Anexo A}

\section{Formato de recolección datos}

Historia según numeración de la clínica de dolor.

Nombre del paciente y acta de junta.

1. Diagnóstico principal en junta medica de algología? (Anotar cie 10 correspondiente)

2. Diagnóstico principal en junta de psicología? (Anotar cie 10 correspondiente)

3. Sexo de paciente: masculino 1 femenino 2

4. Estado socioeconómico: bajo 1 medio 2 alto 3 no hay dato 4

5. Nivel educativo: técnico 1---- universitario 2---- primaria incompleta 3 primaria completa 4---secundaria completa 5 ---secundaria incompleta 6

6. Estado civil: casado 1 ----soltero 2----viudo 3---divorciado 4---unión libre 5---no hay dato 6

7. $\quad$ Eps: arc 1---fac 2---ejc 3---homic 4---otra 5

8. Edad de primera consulta en años

9. Tipo de dolor: somático 1----visceral 2---neuropatico 3

10. Sitio topográfico de dolor (referido por el paciente) Boca 1---torax2--musculo(s) 3----miembro superior4----mano 5----miembros inferiores 6----genitales/region inguinal 7 ----rodilla 8 --- pies/tobillo 9--- hueso/articulaciones 10 ---- cabeza 11---abdomen 12--- cuello 13--- region lumbar 14 ---cintura/pelvis 15--- otros 16 (garganta, ojos, dientes, oido, hemicuerpo, gluteo, perianal) ---hombro 17.

11. Comienzo de dolor: súbito 1---gradual 2

12. Intensidad de dolor por eva al momento de consultar por primera vez en la clínica Ne no entiende ------nd no hay dato ---- el valor numerico

13. Intensidad de dolor por eva posterior a tratamiento Ne no entiende -----nd no hay dato-----el valor numerico

14. Factores modificadores de aumento Actividad física 1 ---cambio de estado de ánimo 2---permanencia en misma posición 3 ---calor 4 ---frio 5---stress 6---otro 7--ninguno 8
15. Factores modificadores de disminución Reposo 1--- cambios de posición 2 ---medicamentos 3---terapia física 4 ---calor 5 ----frio 6 ----ninguna/nada 7----otro 8

16. Alteraciones psicofisiológicas: Sueño 1 --- disminucion en la injesta 2---mareos 3--- nauseas 4--- vomito 5--- estreñimiento 6---- ninguna 7----0tras 8--- sudoración 9----- diarrea 10--- cefalea 11

17. Componente emocional primario: depresión 1 --- ansiedad 2--Rasgos maladaptativos de personalidad 3---stress post traumático 4 ninguno/no se especifica 5

18. Respuesta emocional secundaria Irritabilidad 1---angustia 2---preocupación 3---aislamiento 4----apatía 5--- tristeza 6 --- ansiedad 7 --- temor 8--- no tiene 10

19. Conductas des adaptativas? Abuso de medicamentos 1---inactividad 2----abandono de responsabilidades 3 -ninguna 4

20. Otros factores relacionados a la percepción del dolor Duelo mal elaborado 1---- disfunción de pareja 2--- disfunción familiar 3 -----ausencia de apoyo familiar percibido 4---- antecedentes de abandono en la infancia 6---- sobrecarga de responsabilidades en la infancia 7--- refuerzo familiar 8 ---otros 9 ---- no tiene 10-

21. Alteración en áreas de ajuste Laboral/ocupacional 1--- social 2---familiar 3---afectivo 4---sexual 5---ninguno 6

22. Origen de dolor (según valoración cognositiva del paciente) No traumático 1----traumático no post quirúrgico 2 Traumático post quirúrgico 3

23. Manejo de dolor Farmacológico 1---fisioterapia 2---- bloqueos 3----técnicas espinales 4--- técnicas de estimulación percutánea (tens) 5---- terapia cognitivo conductual 6--- intervención por psiquiatria 7--- intervención por rehabilitación 8--- trabajo social 9---nutrición 10--- reumatología 11--- terapia ocupacional 12---neuropsicologia 13

24. Ocupación Retirado/pensionado 2---- militar 3---- no relacionado a las fuerzas militares/beneficiario 4 ----desempleado 5

25. Tipos de lesión asociado a dolor crónico Cortante 1--punzante 2----corto-contundente 3--- contuso 4----- corto punzante 5----arma de fuego 6-----medios físico - térmicos 7----agentes químicos 8----agentes biológicos 9--psicológicos 10-- no hay dato 11

26. Presenta enfermedad médica crónica diferente a dolor. Si 1 no 2. 